\title{
Acute Liver Failure In Pregnancy
}

\author{
Thomas Byrne* \\ Harlem hospital, 507 Lenox new York, new York 10037.
}

Received: October 08, 2016; Accepted: October 22 2016; Published: December 102016

*Corresponding author: Thomas Byrne ,Harlem hospital, 507 Lenox new York, new York 10037, Email: bordergyn@yahoo.com

\begin{abstract}
Acute liver disease presenting as jaundice is a common event occurring in pregnancy occurring in about 1 per 1000 deliveries. The most common cause of jaundice in pregnancy is viral hepatitis. In the developed world viral hepatitis causes about $2 / 3 \mathrm{~s}$ of cases of jaundice in several series. [1] The great majority of viral hepatitidies are of slow onset and course. They are rarely associated with fulminant liver failure. This is an article about fulminant liver failure in pregnancy. This is extremely rare in Western medicine and there are few references, this is an attempt to develop guidelines to guide diagnosis and treatment.
\end{abstract}

\section{Clinical Case}

This is a collage of several patients and does not exactly represent any particular person or event.

\section{Case One}

A 31 year G4 P3003 presents at 38 weeks with a 3-day history of icterus noticed by her husband but denied by the patient. She also presents with bulbar muscle dysfunction for 12 hours which she has had in the past. She has no other complaints except a decreased appetite for one day. Her pregnancy has been carefully followed because of a history of myasthenia gravis which has been intensively treated in the past and previous auto-immune thyroiditis neither presently active or requiring treatment for a year and a history of moderate to severe asthma which worsened during pregnancy for which she is taking moderate dose inhaled steroids twice a day, singulair once a day and a rescue inhaler required once a week or less. A recent asthma exacerbation required oral steroids 6 weeks before. She has a normally growing fetus with her most recent ultrasound a week ago and has no history of prenatal problems and three normal vaginal births. She has no history of surgical or medical problems but refused thymectomy for her myasthenia and had a recent diagnosis of cholelithiasis found incidentally during a pregnancy ultrasound. She has no history of alcohol, substance, or acetaminophen usage. She has not used any unusual herbs or home remedies. She presents with normal vital signs except for a pulse of 110. She has moderate icterus but no skin jaundice. HEENT exam is normal as are chest and heart exams. Her abdominal exam shows active bowel sounds, no liver tenderness, a soft not-tender uterus with fundal height of 37 centimeters and an unengaged vertex presentation. Her extremities are normal and move normally. Her cervix is $1 \mathrm{~cm}$ dilated soft -2 station with intact membranes. There is no bruising or bleeding from her IV site, gums, or vagina. The FHR, fetal heart rate tracing, is normal. Routine labs for pregnancy and jaundice have been ordered. The bulbar palsy is a sign of worsening myasthenia, neurology is consulted. The neurologist finds minimal but generalized muscle weakness except for bulbar muscles which are moderately weak and recommends resumption of her previous oral corticosteroid. She receives IV corticosteroids because of two corticosteroid treatment courses in the last 6 months The patient's laboratory workup has returned with the WBC is of 13,000 with slight eosinophilia, bands of $5 \%$ and metamyelocytes of $4 \%$, a normal platelet size and morphology with a count of 317,000 , a mild normochromic anemia consistent with her past values and no evidence of DIC, disseminated intravascular coagulation. Her urinalysis is normal, AST is 2435, ALT is 1634. Total bilirubin is 8.5 (NL 0.2 to 1.0 ), direct bilirubin is 2.9 ( $\mathrm{NL} \sim 0$ ). Her albumin is 2.4 grams and $\mathrm{A} / \mathrm{G}$ ratio is 0.6 . Glucose and electrolytes are normal, BUN is 6 , serum creatinine 0.6. Uric acid 4.3. (NL 3.5 to 7.0) Amylase and lipase are normal. Ammonia level 48 (NL 1132). Fibrinogen $120 \mathrm{mg} \%$. PT. 60, corrected INR of 1.5 . PTT is 68 both moderately high, both possibly from reduced fibrinogen production. A urinalysis is normal with no proteinuria and a urobilogen of 0.2 . Two hours later patient develops tachycardia, sweating and change in her mentation over a few minutes. An EKG was normal, a finger stick for glucose was $40 \mathrm{mg} \%$. $5 \%$ dextrose infusion resolved her symptoms within a few minutes. An ultrasound of both her fetus and her liver are performed. The fetal ultrasound is normal with normal estimated fetal weight, normal amniotic fluid, a reactive NST, non-stress test, and normal Doppler of UA, umbilical artery flow, giving a modified biophysical of 4 of 4 . Her liver shows a contracted gallbladder from a meal 2 hours previously but multiple calcified gallstones. Her liver looks normal including size, texture, and bile duct size. There is no evidence of sub capsular hemorrhage, cirrhosis, enlargement or common bile duct obstruction. The Doppler studies of the liver are normal. Internal Medicine and Gastroenterology are consulted to assist with her care. Since she is term there is no reason to delay labor but no immediate need for an immediate cesarean section. 


\section{Case Two}

A 22-year-old G1P0 presents at 37 weeks' gestation with a 4-hour history of severe nausea and vomiting. She also reports reduced fetal movements for 2 days. She has no history of medical or surgical problems. She has had a normal prenatal course with an ultrasound in the last week showing a normally growing singleton fetus. Her only significant past history was an overnight observation 3 weeks ago because of intense RUQ pain nausea and vomiting following a very fatty meal. Her symptoms suggested gallstones as did an AST of 112, an ALT of 240, normal CBC and electrolytes, amylase and lipase, total bilirubin of 0.4 direct of 0.2 . She was given pain relief, hydrated, and observed. A liver ultrasound showed no gallstones or biliary obstruction but a final reading was not available. She was discharged home after her AST dropped to 86 and her ALT to 200 over 6 hours and repeat labs showed no other abnormalities with no further therapy. She was discharged on a low-fat diet. She has no history of drug, alcohol, acetaminophen, or herb usage. Her vital signs are normal except for a blood pressure of 140/89 (up from 100 /69 average during pregnancy) including FHR. She has no jaundice or icterus. HEENT exam is normal as are chest and heart exams. Her abdominal exam shows active bowel sounds, a tender but normal size liver, a soft, non-tender uterus of $37 \mathrm{cms}$ fundal height with an engaged vertex presentation. Her extremities are normal except for $3+$ leg edema. Her cervix is $1 \mathrm{~cm}$ dilated, unsoftened, 0 station, with intact membranes. There is no bruising or bleeding. Routine labs for pregnancy and liver problems are ordered. The patient feels better after IV Phenergan. The laboratory workup has returned. WBC is 7,800 with a normal differential, a normal platelet size and morphology with a count of 5,000, amid normochromic anemia consistent with mild iron deficiency and no evidence of DIC. Her urinalysis shows 3 plus protein. AST is 202 , ALT is 363 Total bilirubin is 0.4 , direct 0.3 . Her albumin is 2.3 with an $\mathrm{A} / \mathrm{G}$ ratio of 0.7 . Glucose and electrolytes are normal. BUN is 7 and creatinine is 0.7 (they were 10 and 0.63 weeks ago). Uric acid is 5.3. Amylase, lipase, and ammonia are all normal Fibrinogen is $350 \mathrm{mg} \%$ Her PT is 20 and her PTT is 12 and her INR is 0.9. An ultrasound of both the uterus and liver were performed. The fetal ultrasound shows a normal fetal weight, amniotic fluid and UA Doppler waveform and a reactive NST, giving a modified biophysical of 4 out of 4 . Her liver looks normal in size with no biliary stones or obstruction. There is no evidence of other abnormalities. The ultrasound reading including review of previous ultrasound shows extensive "fatty liver changes worse than 3 weeks ago", but normal Doppler studies of the liver. She is admitted due to her worsening liver tests and reduced fetal movement at term.

\section{Diagnostic Workup Of New Onset Jaundice}

The initial diagnosis panel for jaundice includes just a few tests. An automated CBC, with microscopic inspection if required, a liver profile including the enzymes AST, aspartate aminotransferase, and ALT, alanine aminotransferase, both direct and indirect bilirubin, glucose and ammonia, a PT1, prothrombin time, PTT, partial thromboplastin time, fibrinogen and albumin levels, a BUN, blood urea nitrogen, serum creatinine, acetaminophen level and a urinalysis. From these tests and a set of vitals a physical exam and history we will be able to start the process of determining the severity of a patient's medical condition, get a start on determining the need for transport and start to identify or at least rule out some of the causes of acute liver failure in a pregnant patient. The other important but easily determined factor is the estimated gestational age either from her prenatal record, her history or a new ultrasound or all three. The immediate health of the neonate at present is determined from its electronic fetal heart surveillance and biophysical profile. Further tests that need to be drawn are antibody and antigen titers for the common platitudes but these will be ordered by your consulting internist or gastroenterologist.

\section{Defining Liver Failure}

I would define liver failure as the inability of the liver to perform its immediate important functions. The most acute of these are maintenance of serum glucose in the fasting state, continuous production of coagulants to maintain clotting activity and removal of metabolic poisons, some of which are probably still unknown, but the most important of which is ammonia. If the liver is failing it is usually failing in all three of these functions. So in a community hospital finding a patient with an elevated ammonia, low fibrinogen and prolonged coagulation profile with hypoglycemia is reason to consider transfer to a larger center. The determination of potential fetal viability is important in two aspects, first several of the acute liver failures of pregnancy only occur after fetal viability and are much more common after 34 weeks of gestation and the finding of fetal viability means that there has to be an immediate analysis of whether delivery in beneficial to either or both the mother and neonate. With HELLP syndrome and AFLP both of which almost universally occur only near term, delivery is beneficial to both the mother and the neonate. Whether delivery is safer for either, before or after transport, is a unique complex decision for each individual patient couple, practitioner, hospital and transport system.

\section{Fulminant Liver Failure}

Fulminant liver failure in pregnancy is much rarer in pregnancy than jaundice and requires aggressive treatments to maximize maternal and fetal outcomes as opposed to new-onset jaundice. A patient will probably present in one of three ways; new onset jaundice, probably scleral, new onset somnolence and unusual mental changes or with hypertension, abdominal pain and bleeding. In the United States by far the most common cause of fulminant liver failure is acetaminophen poisoning.[2]

After diagnosis of fulminant liver failure, the later diagnostic and therapeutic regimens need to be carried out in a center capable of managing all the manifestations and treatments required in this condition. Early transfer to a center with maternal-fetal medicine, neonatology and gastroenterology support and a blood bank capable of large transfusions and specialty products required in this condition is essential. Part of the decision on whether to transfer involves the gestational age and the capability of your hospital to care for a neonate of your patient's gestational age. In some of these causes of fulminant liver failure early delivery is beneficial for the patient and 
possibly the neonate. There are tests required in this condition to quickly allow identification of those disease entities that require expedited delivery. Some tests are to determine the severity of the liver failure, some are to differentiate the cause of a particular patient's condition.

The first group is physical exam and history looking for patient mental acuity and alertness, degree of scleral and skin jaundice, liver and spleen size and tenderness and adenopathy. It is also important to note the patient's pulse, temperature and blood pressure. These are all used in later differentiation of these various disease processes. The history should include alcohol intake, acetaminophen usage, history of previous jaundice, history or family history of autoimmune diseases, immunosuppressive diseases, preeclampsia or liver or metabolic diseases. These should already be obtained from the jaundice workup.

The second group is laboratory tests that help place the patient in a disease category. These are the same labs already drawn for the workup of new onset jaundice. These are hepatic enzymes, direct and indirect bilirubin, serum ammonia, CBC with microscopic inspection if required, fibrinogen concentration, serum amylase and lipase, glucose level, PT, (prothrombin time) and PTT, (partial thromboplastin time), INR (derived by dividing the PT by the average PT for your hospital) and finally serum albumin. These should be available almost immediately. Tests that are important but that will not be immediately available include tests for all hepatic viruses and herpes simplex and their antibodies. Anti-nuclear antigen, ANA, lupus anticoagulant, total immune globulin and anti-liver specific antibodies.

The third group is imaging tests required to diagnose this clinical problem. The first is uterine ultrasound and fetal heart rate testing. Ultrasound liver imaging looking for cholecystitis, cholelithiasis, hepatic duct dilation, Doppler studies of the venous and arterial system of the liver and evidence of cirrhosis or hepatic enlargement or subcapsular hemorrhage. Again these should have already been done for the jaundice workup.

With these tests we should be able to come to a preliminary diagnosis and from the working diagnosis a treatment plan. This of course has to include not only maternal-fetal medicine but internal medicine and gastroenterology but eventually anesthesiology and neonatology. One other important point in managing these disease entities is that serum testing should be repeated after the first 12 hours of admission to determine the course and rapidity (trajectory) of the patient's liver failure.

There are two important causes of fulminant liver failure in pregnancy that need to be ruled out early in a patient's hospital admission because they carry important therapeutic and pregnancy associated risks. The two clinical entities are HELLP syndrome and acute fatty liver of pregnancy. A short list of the most common causes of fulminant liver failure in pregnancy includes AFLP, acute fatty liver of pregnancy, Budd Chiari syndrome, AFLD, alcoholic hepatitis, non-alcoholic hepatitis, HELLP, Hemolysis elevated liver enzymes and low platelets, and autoimmune hepatitis. There are other causes of fulminant liver failure in Europe and North America but are rarer, have more complex testing and differentials and require a gastroenterologist also knowledgeable in their diagnosis and treatment. This is an article to help an Obstetrician start the process of care for an affected pregnant patient. Definitive treatment will require not only a knowledgeable gastroenterologist and internist for help but the services available only in a large referral hospital, such as a large lab with a blood bank capable of providing many units of blood and blood products in an expeditious manner, an intensive care unit with intensivists and a knowledgeable anesthesia department.

\section{Clinical Causes of Fulminant Liver Failure In Pregnancy}

Budd-Chiari Syndrome: This syndrome which is extremely rare involves the change in physiology that results from a thrombotic event involving the veins draining the liver. The presentation is a triad of abdominal pain, ascites and liver enlargement. The disease process involves two processes. The body adaptation to obstruction of blood flow returning through the hepatic bed and the hepatic metabolic derangements that result from this altered blood flow. Although rare pregnancy, pregnancy is the second leading entity associated with BuddChiari. It can present as a chronic condition but in pregnancy appears acutely with the classic triad. [3] In the past the diagnosis was sometimes made in pregnancy by exploratory laparotomy. [4]We then progressed to ultrasound and liver biopsy for diagnosis.[5] Now abdominal ultrasound is usually definitive during the immediate presentation.[6] Of course an MRI is also available for further definition if needed especially in the morbidly obese where there are significant limitations for ultrasound. There are no agreed upon management guidelines in pregnancy specific for treatment of Budd-Chiari.[7] Whether to use anticoagulants or to deliver immediately is not agreed upon.

\section{Hellp Syndrome}

This syndrome is caused by and unique to pregnancy. Its diagnosis involves detection of disseminated intravascular coagulation, elevated liver enzymes and thrombocytopenia in a pregnant patient, in the absence of other causes.[8] Most investigators consider HELLP to be a variant of pre-eclampsia and most patients with HELLP have mild to severe signs of preeclampsia and renal dysfunction.[9] The only cure for HELLP syndrome is delivery. One of the leading institutions in the United States believes that corticosteroids shortens the course and intensity of HELLP syndrome after delivery.[10] Patients usually complain of pain in the area of the liver. Hypertension, even extremely severe hypertension, can be a leading finding and the absence of any blood pressure elevation almost rules out HELLP syndrome as a working diagnosis. It generally presents in the third trimester near term but can present even in the second trimester.[11] The pathognomonic hallmark is disseminated intravascular coagulation and you cannot consider a HELLP syndrome diagnosis without it.[12]

\section{Acute Fatty Liver Of Pregnancy}

This is thought to also be a condition unique to and caused by pregnancy. It occurs almost exclusively only in the late third trimester to post-par tum.[13] There are several world-wide 
individual reports of AFLP in the second trimester. [14,15] Fever is present with jaundice in more than $70 \%$ of cases. [16] Although the exact cause is unknown it is thought to be related to the fetal inheritance of a defective enzyme that catabolizes long chain fatty acids leading to a fetal buildup and then a maternal buildup of these same partially metabolized fatty acids from placental fetal to maternal transfer. The enzyme is called 3-hydroxyacylCoA dehydrogenase. [17] The excess long chain fatty acids are transported in the blood to the liver absorption apparently causes gradually increasing liver dysfunction. [18] The starting manifestations are almost always headache and fatigue. $70 \%$ complain of nausea and vomiting. [19] 80\% complain of left upper quadrant pain. Hepatic encephalopathy will occur late not early in the disease process and itching and a skin rash can occur weeks before intense symptoms begin.[20] This makes some to believe this is an extreme cholestasis of pregnancy.

\section{Alcoholic Fatty Liver Disease}

Autoimmune hepatitis is another cause of jaundice in pregnancy. Although the majority present with chronic slowonset disease $40 \%$ present with acute symptoms. Even in the acute group fulminant hepatic failure is rare. [21] The most common presentation of alcoholic hepatitis during pregnancy is from bleeding varices caused by the venous obstruction of cirrhosis.[22] There are no case reports of acute liver failure in pregnancy caused by alcohol found within the last fifteen years. This despite the reported usage of alcohol to some degree by 15 to $20 \%$ of pregnant patients in the United States.[23] The incidence of cirrhosis is also unknown but thought to be low due to the endocrine effects of reduced liver failure and its effect on fertility.

\section{Non-Alcoholic Fatty Liver Disease}

Nonalcoholic fatty liver disease, NAFLD is the liver manifestation of the metabolic syndrome and is reported to be the commonest liver disease in the world. [24] There is nevertheless little known of its occurrence or symptoms in pregnancy.[25] It is usually a mild disease associated with jaundice but not fulminant liver failure.[26] The disease can occur in any trimester with no known predilection for any. It usually presents with mild jaundice and minimal liver dysfunction. It can progress fulminant failure with jaundice, coagulopathy and coma. [27] Most reports of severe liver failure in pregnancy from fatty liver are in the older literature and most describe disseminated intravascular coagulation which would not be considered part of non-alcoholic fatty liver disease.[28] In the non-pregnant patient most people with NAFLD do no present with symptoms. There are estimates that as many as $20 \%$ of the United States population has NAFLD at this time. [29] The definitive diagnosis is made only by liver biopsy. [30]

There is very little written about this disease entity in pregnancy. The risk of fulminant liver failure from this pathology is higher in older more obese patients with insulin resistance. [31]

\section{Auto-Immune Hepatitis}

Although not thought to be a common cause of acute liver failure in pregnancy, auto-immune hepatitis is perhaps the best described in pregnancy thanks to a seminal work on it published in 2000 of 162 females with this condition and pregnancy. [32] They describe 35 pregnancies resulting in 31 live births including patients that already had cirrhosis. This article found that this form of hepatitis was usually less active during pregnancy and more active in the three months following delivery. They did have 4 acute liver flares in their 35 pregnancies though. It was also interesting that 2 of their cohort of 31 pregnancies after the second trimester developed eclampsia, a rate of 7\%, easily 100 times the usual rate in pregnancy. A group at LSU did a Medline search finding 58 pregnant women with AIH worldwide. They reported a maternal mortality of $4 \%$ and a fetal mortality of about $33 \%$ but counting first trimester losses. All patients including their cases responded to corticosteroids and azathioprine.

\section{Determining An Intial Diagnosis In Acute Liver Failure In Pregnancy}

Let's look at a table that allows a relatively rapid differentiation into the six most important and common causes of acute fulminant liver failure in a pregnant patient. It is important to realize that any of these entities can present or have any kind of lab values, so no single test is diagnostic or rules out any particular entity. It is the overall pattern that is important and sometimes a definitive diagnosis is not possible even with a later liver biopsy. Arguably the only definitive diagnosis of liver failure is acetaminophen poisoning with an elevated level of that toxin. This is also the most treatable early with $\mathrm{N}$-acetyl-cysteine so it is important to not miss that diagnosis by not ordering that lab test. The rest of the diagnoses are less definable.

\section{Treatment of Liver Failure In Pregnancy}

The first decision is to decide on the viability and health of the neonate. For pregnancies below the threshold of viability fetal monitoring is not warranted and consideration of the fetal effects of any required medication should not be considered. Anything life-saving for the mother will be beneficial to the neonate to maximize its chances of achieving viability. Pregnancies clearly in the zone of fetal viability need to have continuous fetal monitoring and the patient delivered, by cesarean section if necessary, to maximize the neonatal life chances and minimize its morbidity. To this end if deficits in the maternal coagulation system are present they need to be corrected in an ongoing fashion to facilitate operative delivery if it is needed in a rapid fashion. For the cases where fetal viability is not yet a reasonable expectation but might be shortly there will be a dilemma about the best interests of the mother and neonate which might differ. In cases of early viability where steroids would be clearly beneficial to the neonate the only relative contraindication in these diseases are with herpetic hepatitis where there is theoretical disadvantage to the mother. Corticosteroids have actually been used in all the other entities sometimes with proven benefit but never with proven harm.

\section{Using Table One}

CASE ONE Let us now use Table 1 to see if a working diagnosis is discernible in case one. The emphasis is on "working diagnosis". Definitive diagnosis requires liver biopsy in most cases and that will not be performed during the pregnancy. She 
Table 1: Differentiating Causes Of Liver Failure In Pregnancy

\begin{tabular}{|c|c|c|c|c|c|c|}
\hline & HELLP & AFLP & Budd-Chiari & AFLD & non-AFLD & Auto-immune \\
\hline Hypertension & $80.00 \%$ & $60.00 \%$ & No & No & No & No \\
\hline Somnolence & No & $60.00 \%$ & Rare & $10.00 \%$ & $10.00 \%$ & $30.00 \%$ \\
\hline Oliguria & $90.00 \%$ & $50.00 \%$ & Rare & No & No & No \\
\hline Proteinuria & $90.00 \%$ & No & No & No & No & No \\
\hline Bleeding & Frequent & Frequent & No & Late & Late & Late \\
\hline Liver pain & $90.00 \%$ & $30.00 \%$ & $20.00 \%$ & No & No & No \\
\hline Vomiting & $50.00 \%$ & $60.00 \%$ & No & No & No & No \\
\hline Hex of jaundice & No & No & No & Yes & Yes & Yes \\
\hline Low platelets & Always $<100 \mathrm{k}$ & possible & possible & No & No & No \\
\hline Eosinophilia & No & No & No & No & No & Yes \\
\hline Renal dysfunction & $90.00 \%$ & $50.00 \%$ & Late unless fulminant & Late & Late & Late \\
\hline WBC count & Normal & Greater than 15,000 & Decreased to less than 500 & Normal & Normal & Normal \\
\hline AST/ALT & Up to 200 & 300 to 500 & $60-400$ & $60-500$ & $60-300$ & $200-2000$ \\
\hline Uric acid & Elevated & Elevated & Normal & Normal & Normal & Normal \\
\hline Fibrinogen & Very low & Mild drop & Mild drop & Mild drop & Mild drop & Very low \\
\hline Ammonia & No & Late & Late & Early & Late & Early \\
\hline Fever & No & $50.00 \%$ & No & No & No & No \\
\hline INR prolongation & Mod-severe & Moderate & Rare & Mod-severe & Moderate & Mod-severe \\
\hline Hx auto-immune $d x$ & Possible & No & Possible & No & No & Yes \\
\hline Low glucose & No & Yes & No & Yes & Yes & Yes \\
\hline Doppler DX & Subcapsular bleed & Normal & Doppler vein changes & Cirrhosis possible & Cirrhosis possible & Cirrhosis possible \\
\hline Bilirubin elevation & Mild & Mild-mod & Mild & Mod-severe & Moderate & Mod-severe \\
\hline Jaundice & Rare & $70.00 \%$ & No & Mod-severe & Moderate & Mod-severe \\
\hline DIC & $100.00 \%$ & $55.00 \%$ & Rare & No & No & No \\
\hline
\end{tabular}

has no evidence of fever, hypertension, DIC, renal dysfunction. Further although her INR is prolonged it is only moderately prolonged suggesting low fibrinogen as the leading cause rather than DIC. She has a normal platelet number and morphology and a reasonably normal CBC. She also has no liver pain or vomiting. Together these rule out HELLP syndrome and AFLP. She has a normal liver arterial and venous Doppler exam which by itself rules out Budd-Chiari syndrome. This leaves us with 3 possible entities causing her fulminant failure in the absence of an elevated acetaminophen level. None of them are helped by expedited delivery but no term fetus is helped by the metabolic environment of a failing maternal liver. The three entities are again; alcoholic fatty liver disease AFLD, non-alcoholic fatty liver disease NAFLD and auto-immune hepatitis. So with this working diagnosis induction of labor is started with oral misoprostol, along with periodic glucose testing, urine output monitoring, the blood bank is called to determine if an adequate supply of freshfrozen plasma, FFP, in her type is available and to cross-match the patient for 4 units of packed irradiated red blood cells, PRBCs and to have the blood and FFP transported to the labor and delivery suite. They are both available within half an hour. Her bulbar palsy is intermittent and a sign of worsening myasthenia, neurology consult is ordered. The neurologist finds minimal but generalized muscle weakness except for bulbar muscles which are moderately weak and recommends resumption of her previous oral corticosteroid. She receives IV corticosteroids because of two corticosteroid treatment courses in the last 6 months. Her bulbar palsy is intermittent and a sign of worsening myasthenia, neurology consult is ordered. The neurologist finds minimal but generalized muscle weakness except for bulbar muscles which are moderately weak and recommends resumption of her previous oral corticosteroid. She receives IV corticosteroids because of two corticosteroid treatment courses in the last 6 months. The fetal heart rate is reactive at present and because she is multiparous she is given oral misoprostol to induce labor and an anesthesia consult is made and $10 \mathrm{ml} / \mathrm{kg}$ of FFP is ordered to normalize her coagulation profile in anticipation of delivery and possible anesthetic or surgical intervention for her delivery. Internal medicine orders a larger battery of tests including tests for viral hepatitidies. At this point her older records are available and they reveal that this patient has had two episodes of jaundice with mild liver dysfunction lasting several weeks and resolving several years ago. At that time, it was thought that auto-immune hepatitis was ruled out although about $20 \%$ of patients with this condition have negative results of tests that are somewhat specific for that entity. A liver biopsy was never performed due to patient reluctance. 
The patient starts a contraction pattern several hours after her misoprostol oral dosage. She later develops a category 2 then a category 3 fetal heart rate tracing. She is given a general anesthetic because of the clotting dysfunction and delivered by cesarean section. Her baby is delivered with low APGAR s from the anesthetics but normal blood gases and $\mathrm{PH}$ and does well in the normal nursery. There were no bleeding issues during her surgery. FFP and one unit of blood are given during the operation to exactly replace losses. Her urine output decreases during the surgery despite exact replacement of fluid losses and she is transported to the labor and delivery intensive care unit. 1500 milliliters of lactated ringers and 500 of $5 \%$ dextrose solution and one more unit of FFP are enough to start to restore adequate urine output. Since she is delivered and has undergone another stress and there is a possibility of this being another auto-immune phenomenon, intravenous steroids are given in a dose usual for auto-immune hepatitis and for treating fatty liver disease failure. She is transported to the medical intensive care unit since she is no longer pregnant. She was at first unresponsive thought to be from poor hepatic drug metabolism. Over the next several hours she becomes more responsive but then has lengthening episodes of somnolence. Her ammonia level rises and her INR rises to 5 and her ammonia level worsens, in the medical ICU it is determined that the patient

has a hidden alcohol abuse problem which may be the cause and/or contribute to her worsening liver failure? She is transported the second day to a liver transplant unit and placed on the list of patients needing a liver transplant. After a week of worsening liver dysfunction, she is placed on the liver transplant list and receives a liver transplant and has slowly recovered from her liver failure over the next few months.

CASE TWO Let us again use table one for a working diagnosis. She has no fever, but has mild hypertension, no evidence of DIC, she has a significant worsening of her renal function in the last 3 weeks, there is no evidence of DIC and her clotting functions are normal. She does have pain over her liver on palpation and her most significant complaint is intense vomiting which has improved with treatment to intense nausea. She also has moderate elevation in her liver enzymes and her bilirubin. Taken together these rule out HELLP syndrome, a normal liver Doppler has ruled out Budd-Chiari. She has intense nausea, hypertension, renal dysfunction, proteinuria, moderate liver enzyme elevation and moderate renal dysfunction. Together these rule out all of the remaining pathologies except AFLP, acute fatty liver of pregnancy. She seems to have only mild disease at present which is unusual but otherwise matches the profile well. She was started on a dextrose drip with periodic glucose testing, oxytocin to initiate labor, and urine output to monitor renal function. She was also started on a magnesium sulfate drip in case this was an unusual preeclampsia presentation. She is typed and crossed for four units of PRBCs but not for other blood components due to her normal fibrinogen and clotting profile. Her blood pressure increases over the next four hours and she is started on IV labetalol and magnesium sulfate. A repeat CBC and liver profile were done. CBC was essentially unchanged, AST has risen to 304 , ALT to 435 total bilirubin to1. 0 and direct to 0.7. A repeat hepatic profile 2 hours later is unchanged except for total bilirubin which has risen to 1,1 and direct to 0.8 She has started a reasonable contraction pattern which the fetus tolerates. After several hours there is fetal intolerance of labor and the patient is delivered by cesarean section under spinal anesthesia. There are no operative or anesthetic problems. She is kept on a magnesium sulfate drip for 24 hours but has no other problems. 24 hours after delivery her BUN and creatinine have improved AST has dropped to 163 ALT to 291 total bilirubin 0.7 direct 0.4 . Her other labs remain normal. By 48 hours she is clinically normal and her AST is 43 and her ALT 138 total bilirubin is 0.4 and direct is 0.2 , BUN is 6 and creatinine is 0.7 both approximately what they were 5 weeks previously.

\section{Conclusion}

These patients demonstrate how using commonly and rapidly available tests and physical exam someone with fulminant liver failure can be properly diagnosed and treated. For the Obstetrician there are two important questions that require answering. Are my hospital and I capable of treating all the possible needs of this patient and her neonate? If the answer to this is doubtful then transport as soon as possible to a center capable of care is required. The second question is to rule out or in and to treat HELLP syndrome and AFLP. In the second case the patient undoubtedly had AFLP but presented in such a mild manner that induction of labor rather than a cesarean section was tried. Although eventually both of these patients required expedited delivery the important action is to have a plan in place for cesarean delivery even when you are trying to facilitate vaginal delivery especially In a stable patient with a category one fetal tracing, being prepared for a cesarean section makes induction of labor safer. A cesarean section increases the odds of excess bleeding and adds anesthetics from general anesthesia to the patient's somnolence, since neuraxial anesthetics are usually ruled out by the coagulopathy of these two conditions.

Once HELLP and AFLP are ruled out, after viability, it is still reasonable to expedite delivery at any time after 34 weeks' gestation. A cesarean section here also complicates the care of the patient after her delivery if she develops fever or has a bleeding problem at her surgical site or uterus. In both scenarios a plan for induction of labor necessarily also means the preparation for a cesarean section should be in place. The table presented in this article should help you develop a sense of how to diagnose these various conditions. The laboratory tests and physical signs are obtainable everywhere. It is important however that although you should develop your own diagnosis that you involve internal medicine, gastroenterology and anesthesia early in the patient's hospital course both for guidance and to inform your colleagues of potential future problems far enough in advance to facilitate the patient's care.

\section{References}

1. Devarbhavi $\mathrm{H}^{1}$, Kremers WK, Dierkhising $\mathrm{R}$, Padmanabhan $\mathrm{L}$. Pregnancy-associated acute liver disease and acute viral hepatitis: Differentiation, course and outcome. J. Hepatol. 2008;49(6):930-935. doi: 10.1016/j.jhep.2008.07.030. 
2. Larson AM, Polson J, Fontana RJ, Davern TJ, Lalani E, Hynan LS, et al. Acute liver failure study group. Acetaminophen-induced acute liver failure: results of a United States multicenter, prospective study. Hepatology 2006;42(6):1364-1372. DOI: 10.1002/hep.20948.

3. Kyriakidis, A.V., Vezyrgiannis, I., Pyrgioti, M. Budd-Chiari syndrome. Ann. Gastroenterol.2004;21(4):223-228.

4. M. Tiliacos, D.Tsantoulas, A.Tsoulias, E.Kokka, E.Eudaimon, S Aphentoglou, et.al. The Budd-Chiari syndrome in pregnancy. Postgrad. Med. J.1978;54(636): 686-691.

5. Fickert P, Ramschak H, Kenner L, Hoefler G, Hinterleitner TA, Petritsch $\mathrm{W}$, et.al. Acute Budd-Chiari syndrome with fulminant heaptic failure in a pregnant women with factor V Leiden mutation. 1996;111(6);16701673.

6. Rautou PE, Angermayr B, Garcia-Pagan JC, Moucari R, PeckRadosavljevic M, Raffa S. et.al. Pregnancy in women with known and treated Budd-Chiar syndrome: maternal and fetal outcomes. J. Hepatol. 2009;51(1):47-54. doi: 10.1016/j.jhep.2009.02.028.

7. Aggarwal N, Suri V, Chopra S, Sikka P, Dhiman RK, Chawla YK. Pregnancy outcome in Budd Chiari Syndrome-A tertiary care centre experience. Arch. Gynecol.Obstet. 2013;288(4):949-952. doi: 10.1007/s00404-013-2834-8.

8. Haram K, Svendsen E, Abilgaard U. The HELLP syndrome: Clinical issues and management. A review. BMC Pregnancy and childbirth .2009;9(8) 1-66. DOI: 10.1186/1471-2393-9-8.

9. Padden M.O. HELLP syndrome: Recognition and perinatal management. Am. Fam. Physician .1999;60(3): 829-836.

10. Wallace K, Martin JN Jr, Tam Tam K, Wallukat G, Dechend R, Lamarca B. Seeking the mechanism(s) of action for corticosteroids in HELLP syndrome: SMASH study. Am.J. Ob. Gyn. 2013;208(5):380.e1-8. doi: 10.1016/j.ajog.2013.01.049.

11. Kelly J, Ryan D.J, O’Brien N.O, Kirwan W.O. Second trimester hepatic rupture in a 35 year old nulliparous woman with HELLP syndrome: a case report. World J. Emer. Surg.2009;4:23. doi: 10.1186/17497922-4-23.

12.Tsokos,M. Pathological features of maternla death from HELLP syndrome.2004;1(1):275-290.

13. Hin Hin Ko , Eric Yoshida. Acute ftty liver of pregnancy. Can. J. Gastroenterol.2006;20(1):25-30.

14. Buytaert IM, Elewaut GP, Van Kets HE. Early occurrence of acute fatty liver in pregnancy. Am. J. Gastroenterol. 1996;91(3):603-604.

15. Monga M., Katz A.R. Acute fatty liver in the second trimester. Obstet. Gynecol. 1999;93(5 PT 2):811-813.

16. Simpson KR, Luppi CJ , O’Brien-Abel N. Acute fatty liver of pregnancy. J.Perinat. Neonatal Nurs. 1998;11(4):35-44.

17.Schoeman,M.N., Baley,R.G., Wilcken,B. Recurrent acute fatty liver of pregnancy associated with afatty-acid oxidation defect in the offspring. Lancet (1991) 100: 544-546.

18.Jamal A Ibdah. Acure fatty liver of pregnancy: an update on pathogenesis and clinical implications. World J. Gastroenterol. 2006;12(46):7397-7404. doi: 10.3748/wjg.v12.i46.7397.
19. Kaplan M.M. Acute fatty liver of pregnancy. N.Eng.J. Med. 1985;313(6):367-370. DOI: 10.1056/NEJM198508083130606.

20. Reyes H, Sandoval L, Wainstein A, Ribalta J, Donoso S, Smok G, et al. Acute fatty liver of pregnancy: a clinical study of 12 episodes in 11 patients. Gut.1994;35(1):101-106.

21.Mann M.P , Czapa J.A ,Gorham J.D , Krawitt E.L , Mieli-Vergani G , Vergani $D$, et al. Diagnosis and management of autoimmune hepatitis. Hepatology .2010;51(6):2193-2213.

22. Puljic A., Salati J., Doss A., Caughey A.B. Outcomes of pregnancy complicated by liver cirrhosis, portal hypertension, or esophageal varices. J. Mat. Fet \& Neonatal Med. 2016:29(3).doi.org/10.3109/147 67058.2015.1009438.

23. US Dept. Health Human Services, Substance Abuse and Mental Health Services Administration, Office of Applied Studies, National Household Survey on Drug Use and Health, 1994. Rockville,Md: US Dept Health Human Services.1995

24. Page,L.M. Girling,J.C. A novel cause for abnormal liver function tests in pregnancy and puerperium- non-alcoholic fatty liver disease- the first reported case series. Arch. Dis.Child Fetal Neonatal. Ed. 2010:95;5960. doi:10.1136/adc.2010.189753.89.

25. Hannes Hagström, Jonas Höijer, Jonas F. Ludvigsson, Matteo Bottai, Anders Ekbom, Rolf Hultcrantz . Adverse outcome of pregnancy in women with non-alcoholic fatty liver disease. Liver Int. 2016;36(2):268-274.doi: 10.1111/liv.12902

26.Castracane.v ， Davis,G, Martinez,B, Maguire,C ,Kauffman. R. Nonalcoholic fatty liver disease in pregnant women. Endo. Abtstracts. 2012;29:938.

27. Ockner SA, Brunt EM, Cohn SM, Krul ES, Hanto DW, Peters MG. Fulminant liver failure cuased by acute fatty liver of pregnancy treated by orthotopic liver transplantation. Hepatology. 1990;11(1):59-64.

28.Castro,M.A., Fassett,M.J., Reynolds,T.B., Shaw,K.J., Goodwin,T.M. Reversible peripartum liver failure: a new perepsective on the diagnosis, treatment, and cause of acute fatty liver of pregnancy, based on 28 consecutive cases. Am. J. Obstet.Gynecol. 1999;181(2):389-395.

29. Lazo M.,Clark JM. The epidemiology of nonalcoholic fatty liver diease: a global perspective. Semin. Liver Dis. 2008;28(4):339-350. doi: 10.1055/s-0028-1091978.

30. Clark JM, Brancati FL, Diehl AM. Nonalcoholic fatty liver disease. Gastroenterol. 2002;122(6):1649-1657.

31. Rinella ME. Nonalcoholic fatty liver disease. A systematic review. JAMA. 2015;313(22):2263-2273. doi: 10.1001/jama.2015.5370.

32. Heneghan MA, Norris SM., O'Grady JG, Harrison PM., McFarlane I.G. Management and outcome of pregnancy in autoimmune hepatitis. Gut. 2001 48(1):97-102. doi: 10.1136/gut.48.1.97.

33. Candia L, Marquez J, Espinoza LR. Autoimmune hepatitis and pregnancy: A Rheumatologist's dilemna. Sem. Arthrtis Rheum. (2005) 35(1): 49-56. DOI:10.1016/j.semarthrit.2005.03.002. 